\title{
Demographic, Socio-economic and Lifestyle Determinants of Under- and Over-nutrition among Bangladeshi Adult Population: Results from a Large Cross-Sectional Study
}

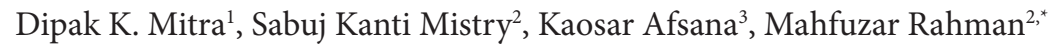 \\ ${ }^{1}$ School of Public Health, North South University, Bangladesh \\ ${ }^{2}$ Research and Evaluation Division, BRAC, BRAC Centre, 75 Mohakhali, Dhaka 1212, Bangladesh \\ ${ }^{3}$ Health Nutrition and Population Programme, BRAC, BRAC Centre, 75 Mohakhali, Dhaka 1212, Bangladesh
}

\section{ARTICLE INFO}

Article History

Received 20 November 2017

Accepted 31 March 2018

Keywords

Underweight

overweight

nutritional transition

factors

\begin{abstract}
Bangladesh is currently going through a nutritional transition with rapid increase in overnutrition while undernutrition is still remaining prevalent. Nevertheless, population-based data on demographic, socio-economic and lifestyle factors associated with underweight and overweight among adult population is scarce. Employing a nationwide cross-sectional survey, we collected anthropometric, demographic, socio-economic, lifestyle and dietary information from 12,180 adults aged $\geq 35$ years. Body Mass Index (BMI) was calculated using standard formula and categorized into underweight $(<18.50)$, normal weight $(18.50-$ $22.99)$, and overweight $(\geq 23.00)$. Multivariable multinomial logistic regression was performed to identify factors associated with underweight and overweight. Overall, prevalence of underweight and overweight was $18.1 \%$ (95\% CI: 17.5-18.8) and 33.7\% (95\% CI: 32.9-34.6), respectively. All the demographic, socio-economic, dietary and lifestyle factors showed significant association with nutritional status in bivariate analysis. In adjusted analysis, factors showing significant positive association with underweight included female gender (ARRR-1.38, 95\% CI: 1.11-1.71), older age [compared to 35-39 years age group, ARRR (95\% CI) for $\geq 70$ years is 2.32 (1.89-2.86), for 60-69 years is 1.62 (1.36-1.93), for 50-59 years $1.34(1.13-1.58)$ and for $40-49$ years $1.05(0.87-1.15)$ ] and smoking habit (ARRR-1.32, 95\% CI: 1.14-1.52) while factors showing significant inverse association with underweight included higher household wealth [compared to lowest wealth quintile, ARRR (95\% CI) for highest quintile is $0.68(0.55-0.84)$, for second highest quintile $0.77(0.65-0.91)$, for middle quintile $0.81(0.69-0.94)$ and for second lowest quintile $0.89(0.77-1.03)$ ], urban residence (ARRR-0.66, 95\% CI: 0.66-0.90), and more frequent meat/fish and fruits consumption (ARRR-0.76, 95\% CI: 0.65-0.90). On the other hand, factors significantly associated with increased risk of overweight included female gender (ARRR-1.35, 95\% CI: 1.12-1.63), higher household wealth [compared to lowest wealth quintile, ARRR (95\% CI) for highest quintile is 2.27 (1.93-2.68), for second highest quintile 1.67 (1.44-1.94), for middle quintile 1.26 (1.10-1.46) and for second lowest quintile $1.07(0.93-1.24)$, excess food availability [compared to food shortage, ARRR (95\% CI) for excess food in the household is $1.29(1.12-1.47)$ and for no shortage/no excess is $1.23(1.09-1.38)$ and more frequent fruits consumption [compared to no fruits, ARRR (95\% CI) for 5-7 days per week consumption is 1.61 (1.41-1.83) and for 3-4 days per week is 1.28 (1.16-1.41) and factors significantly associated with decreased risk of overweight included older age [compared to 35-39 years age group, ARRR $(95 \% \mathrm{CI})$ for $\geq 70$ years is $0.77(0.64-0.93)$, for $60-69$ years is $0.82(0.71-0.94)$, for $50-59$ years $0.91(0.80-1.04)$ and for 40-49 years $1.01(0.89-1.15)$ ] and smoking (ARRR-0.76, 95\% CI: 0.68-0.86). Both underweight and overweight are prevalent in Bangladeshi adult population. Several demographic, socio-economic, dietary and lifestyle factors are associated with underweight and overweight in Bangladesh. Population level impact of these factors should be examined to design suitable public health and nutrition interventions to address this dual challenge.
\end{abstract}

(C) 2018 Atlantis Press International B.V.

This is an open access article under the CC BY-NC license (http://creativecommons.org/licences/by-nc/4.0/).

\section{INTRODUCTION}

Bangladesh like many other developing countries is currently going through a nutritional transition with rapid increase in overnutrition while undernutrition is still remaining prevalent $[1,2]$. Recent demographic and health survey (DHS) shows that prevalence of underweight $(\mathrm{BMI}<18.5)$ in women of reproductive age decreased from $45 \%$ in 2000 to $24 \%$ in 2011 , when overweight $(\mathrm{BMI} \geq 25)$ increased from $5 \%$ to $17 \%$ [3]. Another study

* Corresponding author.Email: mahfuzar.rahman@brac.net carried out among adult people aged 35 years or more, reported $29.1 \%$ and $20.5 \%$ prevalence of underweight and overweight $(\mathrm{BMI} \geq 23)$ respectively in men and $36.0 \%$ and $24.4 \%$ prevalence of underweight and overweight respectively in women in Bangladesh [4].

Adverse health consequences of both undernutrition and overnutrition in adult population are well documented that includes increased mortality and morbidity [5,6]. Underweight is linked with several co-morbidities including osteoporosis, infertility and asthma $[7,8]$, while overweight is associated with cardiovascular diseases, cancer, hypertension and diabetes $[9,10]$. 
Studies in developed countries have extensively examined associations of socio-demographic and lifestyle factors with underweight and overweight [11-14]. However, very few studies in Bangladesh and other South Asian countries have studied factors associated with overweight and underweight [15]. A recent study in Bangladesh reported older age, lower education level and poverty are positively associated with underweight while younger age, female gender, higher education level, affluence and urban residence are positively associated with overweight [4]. An study conducted in adult males in Sri Lanka showed that older age, higher family income and higher education level are positively associated with overweight and obesity [15]. Another study in India among women showed that lower socioeconomic status increased the risk of being underweight while higher socioeconomic status increased the risk of being overweight [16]. Identification of modifiable lifestyle factors with nutritional status from population-based studies is critically important to design public health interventions to prevent impeding epidemic of overnutrition in low and middle income countries. Thus, in present study, we estimated the prevalence of underweight and overweight and their associations with several demographic, socio-economic and lifestyle variables in a nationally representative adult population including both males and females.

\section{METHODS}

\subsection{Study Design}

This is a nationally representative population-based cross-sectional study conducted by the BRAC Research and Evaluation Division (RED) in 2015. The primary purpose of the study was to establish a baseline for the BRAC's nationwide Health, Nutrition and Population Program (HNPP). The larger study included 60,503 respondents including men, women and children of all ages. This study used data from 12,180 adult population of age 35 years or more who provided data on anthropometry, socio-demographic, economic and lifestyle variables.

\subsection{Study Setting and Population}

This study was conducted in 58 of 64 districts of Bangladesh. Households were selected for the survey using multistage cluster sampling approach. Data were collected from all selected households and its members after informed consent was obtained. Household members who were 35 or more years of age were asked for anthropometry and other lifestyle information.

\subsection{Data Collection Tools and Techniques}

The survey questionnaire was field-tested before using for final data collection. All data were collected online in 7 " android tablets using ODK (Open Data Kit), an android based open-source mobile platform software [17]. Trained and experienced interviewers collected detailed data on socioeconomic and demographic characteristics includes age, sex, education, marital status, household wealth and income from the study population. They also measured participants' anthropometry (height and weight), behavioral and lifestyle activities including sleeping duration (in hours), smoking and smokeless tobacco consumption, reported level of physical activity (exercise) and dietary habit.

\section{OUTCOME AND EXPOSURE ASSESSMENT}

Body Mass Index (BMI) was our primary outcome of interest. Our trained data collectors measured the height and weight of all consented respondents using standardized procedure. Weight was measured using digital bathroom scale while height was measured using locally made stadiometer. Digital bathroom scales are recommended for measuring weights for public health research [18] and has been used before [19]. Each respondent was measured twice and an average of the two measurements was considered in the analysis. BMI was calculated using the formula weight in $\mathrm{kg} / \mathrm{height}$ in meter $^{2}$ and classification was done using WHO recommended Asian specific cut points of underweight $(\mathrm{BMI}<18.5)$ and overweight $\geq 23.0$ [20]. Selfreported exposure data were collected from the respondents using a pre-tested questionnaire. Household characteristics were validated with observation by the data collectors. Total sleep time (TST) was defined as "the difference between the time at which the participant switches off the light and the time at which they wake up, ignoring the time needed to fall asleep and the time to wake up" [21]. Each participant was asked to recall his or her TST in the previous $24 \mathrm{~h}$ on the day of interview regardless of daytime or nighttime sleep. As documented in our earlier paper, they were then given sufficient time to calculate their total sleep duration (in hours) for both the day and night [22]. We further categorized sleep duration into $<7 \mathrm{~h}, 7-9 \mathrm{~h}$, and $\geq 10 \mathrm{~h}$ according to National Sleep Foundation guidelines [23].

\subsection{Training and Quality Assurance}

We recruited a total of 110 interviewers with previous experience of online data collection. We organized fifteen-day (2 weeks) intensive training which included lectures, mock interviews, role play and field practice at the community level. All data collectors were standardized on anthropometry procedures. Weighing machines were calibrated weekly to ensure accurate measurements throughout the survey period. We divided data collectors into 21 teams and each team was supervised by an experienced field supervisor. The investigators also frequently travelled to the field to oversee the data collection procedures. Supervisors performed spot checking in the field and cross-checked filled in data in the tab before sending to the BRAC information and communications technology (ICT) server using mobile internet. Around 5 percent of the sampled respondents were re-interviewed by the field supervisors and immediately sent to the headquarters for cross-checking and feedback to the field. In case any discrepancies were found, the field coordinator investigated the issue and rectified. Data were collected during the months of August through December 2015.

\subsection{Statistical Analyses}

We computed means and standard deviation of height, weight and BMI for men and women separately. Prevalence of under-weight (BMI < 18.5), over-weight/obese (BMI $\geq 23.0)$ and normal weight 
(BMI 18.50-22.99) with corresponding 95\% Confidence Interval was calculated by gender and for all respondents. Percent distribution of demographic, socio-economic and lifestyle factors are compared among nutritional categories using chi squared test of independence. Adjusted associations of demographic, socio-demographic and lifestyle factors with underweight and overweight compared to normal weight were examined using multinomial logistic regression. For measuring socio-economic status of households, a wealth score was created using information on ownership of durable household assets, source of drinking water, type of latrine, and characteristics of dwelling house using principal component analysis [24]. The respondents were then grouped into five quintiles based on the ranking of the households according to the wealth score. Data analyses were done in Stata (version 12) statistical software [25].

\subsection{Ethics Statement}

Ethical approval was obtained from the Bangladesh Medical Research Council (Ref: BMRC/NREC/2013-2016/802). The purpose of the study was described to the participants. Both verbal and informed written consent were provided by participants prior to interview.

\section{RESULTS}

A total of 60,503 people in 11,515 households were enumerated by the data collection teams (Fig. 1), of which 15,297 (25.3\%) were $\geq 35$ years old and were eligible for this study. A total of 12,180 (79.6\%) eligible respondents provided all data and were included in the study, of which 5885 (48.3\%) were male and 6295 (51.7\%) were females.

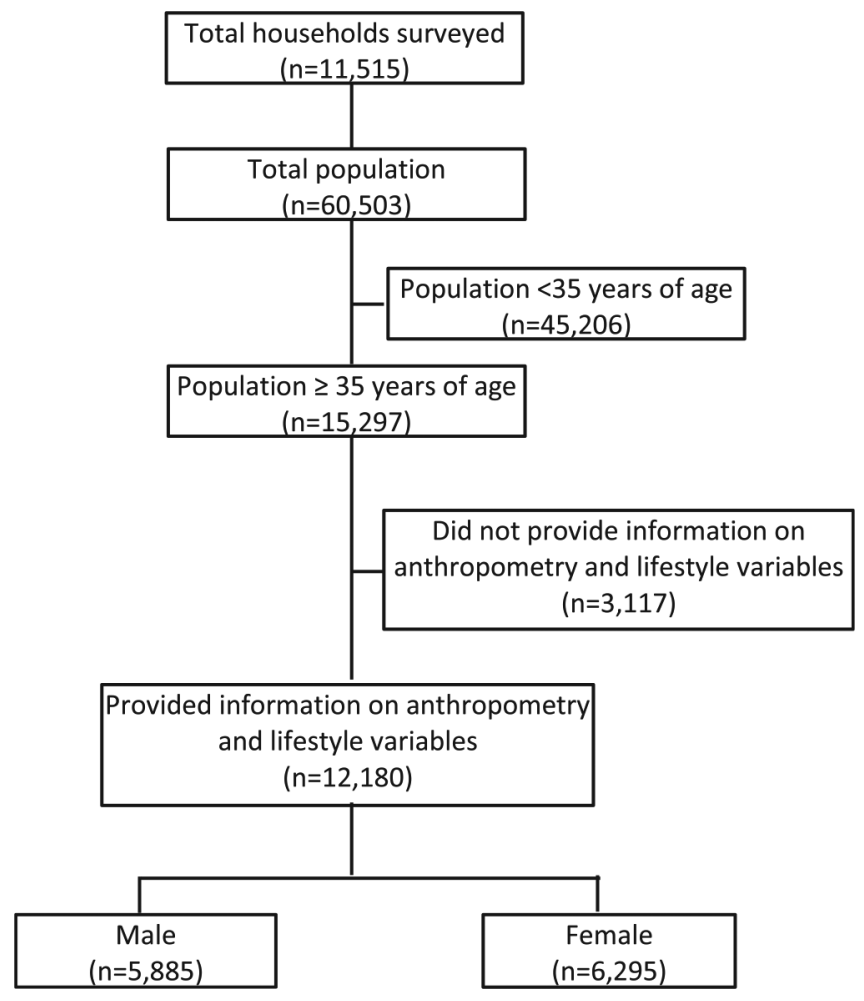

Figure 1 Study profile
The remaining 3117 eligible respondents were either absent during the data collection period (3000) or did not provide consent (117).

Table 1 shows the means and standard deviations of height, weight and BMI scores for all respondents and for men and women separately. Table 2 shows prevalence and corresponding 95\% CI of underweight and overweight in men and women separately and for the total respondents. Overall prevalence (95\% CI) of underweight is $18.14 \%(17.47 \%-18.84 \%)$ and that of overweight (BMI $\geq 23.00)$ is $33.74 \%(32.90 \%-34.58 \%)$. Prevalence of overweight using cutpoints of 25 (International standard) is $18.30 \%(17.62 \%-19.00 \%)$.

Table 3 shows the percent distribution of BMI categories (underweight, overweight and normal weight) by demographic and socio-economic factors (gender, household weight quintile, food availability in the preceding year, age, education level, marital status, occupation and area of residence). All these factors are significantly associated with nutritional status. Prevalence of underweight is found to be higher in females; respondents in lower wealth quintile households; respondents in households reporting food shortage; older people and rural population. While prevalence of overweight is found to be higher in females; respondents in higher wealth quintile households; respondents in households with excess food; younger people; and urban population. Table 4 shows the distribution of nutritional status by lifestyle factors and dietary habits. All lifestyle and dietary factors (protein consumption; vegetable consumptions; fruits consumption; extra salt intake; daily sleep duration; smoking; smokeless tobacco consumption; and exercise) are significantly associated with nutritional status in the surveyed population. Prevalence of underweight is higher among respondents consuming meat/fish less frequently; respondents consuming vegetables more frequently; and respondents consuming fruits less frequently. Prevalence of overweight is higher among respondents consuming meat/fish more frequently; respondents consuming vegetables less frequently; and respondents consuming fruits more frequently.

Table 5 shows the unadjusted and adjusted association of demographic, socioeconomic, lifestyle and dietary factors with underweight and overweight compared to normal weight using multinomial logistic regression. Female gender, older age, and smoking habit are positively associated with underweight in our population. Adjusted Relative Risk Ratio (ARRR) and 95\% confidence interval of underweight for females is 1.38 (1.11-1.71) compared to males. Ever smokers have $32 \%$ higher risk of being underweight (ARRR1.32, 95\% CI: 1.14-1.52) compared to never smokers. Compared to 35-39 years of age group, ARRR (95\% CI) for respondents in 70 years and more group is 2.32 (1.89-2.86), for 60-69 years age group is $1.62(1.36-1.93)$, and for $50-59$ years age group is $1.34(1.13-1.58)$. Higher household wealth quintile, urban residence, weekly 5-7 days meat/fish consumption, and weekly 3-7 days fruits consumption are

Table 1 Descriptive statistics of anthropometric variables by gender among the study population

\begin{tabular}{|c|c|c|c|c|c|c|}
\hline \multirow{2}{*}{$\begin{array}{l}\text { Anthropometric } \\
\text { variables }\end{array}$} & \multicolumn{2}{|c|}{$\begin{array}{c}\text { Men } \\
(\mathrm{n}=5885)\end{array}$} & \multicolumn{2}{|c|}{$\begin{array}{l}\text { Women } \\
(n=6295)\end{array}$} & \multicolumn{2}{|c|}{$\begin{array}{c}\text { Total } \\
(\mathrm{n}=12180)\end{array}$} \\
\hline & Mean & $\begin{array}{l}\text { Standard } \\
\text { deviation }\end{array}$ & Mean & $\begin{array}{l}\text { Standard } \\
\text { deviation }\end{array}$ & Mean & $\begin{array}{l}\text { Standard } \\
\text { deviation }\end{array}$ \\
\hline Height $(\mathrm{cm})$ & 159.58 & 6.43 & 148.82 & 5.80 & 154.02 & 8.14 \\
\hline Weight (kg) & 55.04 & 9.30 & 48.93 & 9.65 & 51.88 & 9.96 \\
\hline BMI $\left(\mathrm{kg} / \mathrm{m}^{2}\right)$ & 21.59 & 3.29 & 22.05 & 3.96 & 21.83 & 3.66 \\
\hline
\end{tabular}


Table 2 Prevalence of under- and over-nutrition according to BMI cut-offs as recommended for Asian population by WHO

\begin{tabular}{|c|c|c|c|c|c|c|}
\hline \multirow{2}{*}{ Nutritional status } & \multicolumn{2}{|c|}{$\operatorname{Men}(n=5885)$} & \multicolumn{2}{|c|}{ Women $(n=6295)$} & \multicolumn{2}{|c|}{ Total $(n=12180)$} \\
\hline & Percent & $95 \% \mathrm{CI}$ & Percent & $95 \% \mathrm{CI}$ & Percent & $95 \% \mathrm{CI}$ \\
\hline Normal weight (BMI 18.5-22.99) & 52.10 & $50.82-53.37$ & 44.40 & $43.18-45.63$ & 48.12 & $47.23-49.01$ \\
\hline Underweight (BMI < 18.5) & 17.25 & $16.30-18.23$ & 18.98 & $18.03-19.97$ & 18.14 & $17.47-18.84$ \\
\hline Overweight/obese $(\mathrm{BMI} \geq 23)$ & 30.65 & $29.49-31.84$ & 36.62 & $35.43-37.81$ & 33.74 & $32.90-34.58$ \\
\hline Over-weight/obese (BMI $\geq 25)$ & 14.46 & $13.58-15.38$ & 21.89 & $20.89-22.93$ & 18.30 & $17.62-19.00$ \\
\hline
\end{tabular}

Table 3 Association of demographic and socio-economic variables with nutritional status of the study population

\begin{tabular}{|c|c|c|c|c|c|c|c|}
\hline \multirow{2}{*}{ Variables } & \multicolumn{2}{|c|}{ Normal weight $(n=5861)$} & \multicolumn{2}{|c|}{ Under-weight $(\mathrm{n}=\mathbf{2 2 1 0})$} & \multicolumn{2}{|c|}{ Over-weight (4109) } & \multirow{2}{*}{ P Value } \\
\hline & Percent & $95 \% \mathrm{CI}$ & Percent & $95 \% \mathrm{CI}$ & Percent & $95 \% \mathrm{CI}$ & \\
\hline \multicolumn{8}{|l|}{ Gender } \\
\hline Male & 52.1 & $50.8-53.4$ & 17.2 & $16.3-18.2$ & 30.7 & $29.5-31.8$ & $<0.001$ \\
\hline Female & 44.4 & $43.2-45.6$ & 19.0 & $18.0-20.0$ & 36.6 & $35.4-37.8$ & \\
\hline \multicolumn{8}{|l|}{ Wealth quantile } \\
\hline Lowest & 53.3 & $51.3-55.2$ & 26.0 & $24.3-27.8$ & 20.7 & $19.1-22.4$ & $<0.001$ \\
\hline Second lowest & 52.7 & $50.7-54.6$ & 21.8 & $20.2-23.5$ & 25.5 & $23.8-27.3$ & \\
\hline Middle & 50.4 & $48.5-$ & 18.6 & $17.1-20.2$ & 30.9 & $29.2-32.8$ & \\
\hline Second highest & 45.5 & $43.6-47.5$ & 15.0 & $13.6-16.5$ & 39.5 & $37.6-41.5$ & \\
\hline Highest & 38.3 & $36.3-40.3$ & 8.9 & $07.9-10.2$ & 52.8 & $50.8-54.8$ & \\
\hline \multicolumn{8}{|l|}{ Food availability in the last year } \\
\hline Always/sometimes shortage & 53.9 & $52.1-55.7$ & 23.3 & $21.8-24.9$ & 22.8 & $21.3-24.4$ & $<0.001$ \\
\hline No shortage/No excess & 47.9 & $46.7-49.3$ & 17.8 & $16.8-18.8$ & 34.3 & $33.1-35.5$ & \\
\hline Excess & 43.7 & $42.1-43.3$ & 14.6 & $13.5-15.8$ & 41.7 & $40.1-43.3$ & \\
\hline \multicolumn{8}{|l|}{ Age } \\
\hline $35-39$ years & 48.8 & $46.9-50.7$ & 12.5 & $11.3-13.8$ & 38.7 & $36.9-40.6$ & $<0.001$ \\
\hline $40-49$ years & 48.7 & $46.9-50.6$ & 13.9 & $12.7-15.3$ & 37.4 & $35.6-39.2$ & \\
\hline $50-59$ years & 47.9 & $46.0-49.7$ & 18.2 & $16.8-19.7$ & 33.9 & $32.2-35.7$ & \\
\hline $60-69$ years & 48.6 & $46.7-50.6$ & 22.3 & $20.7-23.9$ & 29.1 & $27.3-30.9$ & \\
\hline 70 years $\&$ more & 45.2 & $42.6-47.8$ & 30.1 & $27.8-32.6$ & 24.7 & $22.5-27.0$ & \\
\hline \multicolumn{8}{|l|}{ Education } \\
\hline No education & 49.2 & $47.2-51.2$ & 20.3 & $18.7-21.9$ & 30.5 & $28.7-32.4$ & $<0.001$ \\
\hline $1-4$ years of schooling & 49.9 & $47.4-52.3$ & 14.2 & $12.6-16.0$ & 35.9 & $33.6-38.3$ & \\
\hline $5-9$ years of schooling & 43.4 & $41.6-45.3$ & 11.4 & $10.3-12.7$ & 45.2 & $43.3-47.0$ & \\
\hline$\geq 10$ years of schooling & 49.5 & $48.2-50.9$ & 21.8 & $20.7-22.9$ & 28.7 & $27.5-29.9$ & \\
\hline \multicolumn{8}{|l|}{ Marital status } \\
\hline Currently married & 48.3 & $47.3-49.2$ & 16.8 & $16.1-17.6$ & 34.9 & $34.0-35.9$ & $<0.001$ \\
\hline Currently single & 47.5 & $45.4-49.6$ & 24.3 & $22.6-26.2$ & 28.2 & $26.3-30.1$ & \\
\hline \multicolumn{8}{|l|}{ Occupation } \\
\hline Agriculture & 54.6 & $52.1-57.1$ & 21.0 & $19.0-23.2$ & 24.3 & $22.2-26.6$ & $<0.001$ \\
\hline Formal job & 46.5 & $43.1-50.0$ & 9.6 & $07.8-11.8$ & 43.9 & $40.5-47.3$ & \\
\hline Business & 45.0 & $42.3-47.7$ & 9.2 & $07.8-10.9$ & 45.8 & $43.1-48.5$ & \\
\hline Laborer & 62.8 & $59.9-65.6$ & 20.6 & $18.4-23.1$ & 16.6 & $14.5-18.9$ & \\
\hline Home maker & 44.4 & $43.0-45.7$ & 17.8 & $16.8-18.8$ & 37.8 & $36.5-39.1$ & \\
\hline Unemployed & 47.6 & $43.6-51.6$ & 22.4 & $19.3-26.0$ & 30.0 & $26.4-33.8$ & \\
\hline Others & 48.1 & $45.5-50.6$ & 25.6 & $23.4-27.8$ & 26.4 & $24.2-28.7$ & \\
\hline \multicolumn{8}{|l|}{ Residence } \\
\hline Rural & 48.4 & $47.4-49.3$ & 19.2 & $18.4-19.9$ & 32.5 & $31.6-33.3$ & $<0.001$ \\
\hline Urban & 45.8 & $42.9-48.6$ & 8.6 & $07.1-10.4$ & 45.6 & $42.8-48.5$ & \\
\hline
\end{tabular}

inversely associated with underweight. Compared to respondents in the lowest wealth quintile, ARRR $(95 \% \mathrm{CI})$ for respondents in the highest wealth quintile is $0.68(0.55-0.84)$; for respondents in the second highest quintile is $0.77(0.65-0.91)$; for middle quintile is 0.81 (0.69-0.94); and for second lowest quintile is 0.89 (0.77-1.03). Urban respondents have $24 \%$ lower risk of being underweight (ARRR-0.76, 95\% CI: 0.65-0.90). Female gender, higher household wealth quintile, excess food availability, marital status and more frequent fruits consumption are positively associated with overweight; while older age and smoking habit are negatively associated with overweight.
ARRR (95\% CI) of overweight for females is 1.35 (1.12-1.63) compared to males. Compared to respondents in the lowest wealth quintile households, ARRR (95\% CI) for respondents in the highest wealth quintile household is 2.27 (1.93-2.68); for second highest quintile is 1.67 (1.44-1.94); for middle wealth quintile 1.26 (1.10-1.46); and for second lowest wealth quintile 1.07 (0.93-1.24). Respondents reporting excess of food have 29\% higher risk of being overweight (ARRR-1.29, 95\% CI: 1.12-1.47) compared to respondents who reported food shortage in the preceding year. 
Table 4 Association of lifestyle variables with nutritional status of the study population

\begin{tabular}{|c|c|c|c|c|c|c|c|}
\hline \multirow{2}{*}{ Variables } & \multicolumn{2}{|c|}{ Normal weight $(n=5861)$} & \multicolumn{2}{|c|}{ Under-weight $(\mathrm{n}=2210)$} & \multicolumn{2}{|c|}{ Over-weight $(n=4109)$} & \multirow{2}{*}{ P Value } \\
\hline & Percent & $95 \% \mathrm{CI}$ & Percent & $95 \% \mathrm{CI}$ & Percent & $95 \% \mathrm{CI}$ & \\
\hline \multicolumn{8}{|l|}{ Meat/Fish consumption } \\
\hline $0-2$ days per week & 51.2 & $48.5-53.8$ & 26.1 & $23.9-28.5$ & 22.7 & $20.6-25.0$ & $<0.001$ \\
\hline 3-4 days per week & 51.3 & $49.7-52.9$ & 21.7 & $20.4-23.0$ & 27.0 & $25.6-28.4$ & \\
\hline 5-7 days per week & 45.7 & $44.6-46.9$ & 14.6 & $13.8-15.5$ & 39.6 & $38.5-40.8$ & \\
\hline \multicolumn{8}{|l|}{ Vegetable consumption } \\
\hline Consume daily & 51.8 & $49.8-53.8$ & 22.9 & $21.2-24.6$ & 25.3 & $23.6-27.1$ & $<0.001$ \\
\hline Does not consume daily & 47.2 & $46.2-48.2$ & 17.0 & $16.3-17.8$ & 35.8 & $34.8-36.7$ & \\
\hline \multicolumn{8}{|l|}{ Fruits consumption } \\
\hline 0 days per week & 51.9 & $50.6-53.1$ & 22.6 & $21.5-23.7$ & 25.6 & $24.4-26.7$ & $<0.001$ \\
\hline $1-2$ days per week & 47.0 & $45.5-48.5$ & 15.8 & $14.7-16.9$ & 37.2 & $35.8-38.7$ & \\
\hline 3-7 days per week & 39.8 & $37.7-42.0$ & 10.6 & $09.3-12.0$ & 49.6 & $47.4-51.8$ & \\
\hline \multicolumn{8}{|l|}{ Extra salt intake } \\
\hline Never & 46.4 & $44.3-48.5$ & 16.9 & $15.4-18.5$ & 36.7 & $34.7-38.8$ & 0.018 \\
\hline Sometimes & 48.0 & $46.5-49.5$ & 18.3 & $17.2-19.5$ & 33.6 & $32.2-35.1$ & \\
\hline Always & 48.8 & $47.6-50.1$ & 18.5 & $17.5-19.5$ & 32.7 & $31.5-33.9$ & \\
\hline \multicolumn{8}{|l|}{ Daily sleep hours } \\
\hline Less than $7 \mathrm{~h}$ & 47.5 & $44.9-50.0$ & 17.1 & $15.3-19.1$ & 35.4 & $33.0-37.9$ & 0.002 \\
\hline $7-9 \mathrm{~h}$ & 48.9 & $47.8-49.9$ & 18.3 & $17.5-19.1$ & 32.8 & $31.9-33.8$ & \\
\hline More than $9 \mathrm{~h}$ & 44.2 & $41.7-46.7$ & 18.2 & $16.3-20.2$ & 37.7 & $35.2-40.2$ & \\
\hline \multicolumn{8}{|l|}{ Smoking habit } \\
\hline Never smoker & 45.6 & $44.5-46.6$ & 17.1 & $16.3-17.9$ & 37.3 & $36.3-38.4$ & $<0.001$ \\
\hline Ever smoker & 53.6 & $52.0-55.1$ & 20.3 & $19.1-21.6$ & 26.1 & $24.7-27.5$ & \\
\hline \multicolumn{8}{|l|}{ Smokeless tobacco use } \\
\hline Never user & 46.6 & $45.0-48.1$ & 14.9 & $13.8-16.1$ & 38.5 & $37.0-40.1$ & $<0.001$ \\
\hline Ever user & 48.8 & $47.8-49.9$ & 19.7 & $18.8-20.5$ & 31.5 & $30.5-32.5$ & \\
\hline \multicolumn{8}{|l|}{ Exercise } \\
\hline No exercise & 46.3 & $44.6-47.9$ & 20.1 & $18.8-21.5$ & 33.6 & $32.0-35.2$ & $<0.001$ \\
\hline$\leq 3 \mathrm{~h}$ exercise per week & 47.9 & $46.7-49.1$ & 16.9 & $16.1-17.9$ & 35.1 & $34.0-36.3$ & \\
\hline$>3 \mathrm{~h}$ exercise per week & 51.8 & $49.7-54.0$ & 18.7 & $17.1-20.4$ & 29.5 & $27.5-31.5$ & \\
\hline
\end{tabular}

Table 5 Demographic, Socio-economic and Lifestyle factors associated with under- and over-weight in study population

\begin{tabular}{|c|c|c|c|c|}
\hline & \multicolumn{2}{|c|}{ Unadjusted analysis } & \multicolumn{2}{|c|}{ Adjusted analysis } \\
\hline & $\begin{array}{l}\text { Under-weight } \\
\text { RRR (95\% CI) }\end{array}$ & $\begin{array}{c}\text { Over-weight } \\
\text { RRR (95\% CI) }\end{array}$ & $\begin{array}{l}\text { Under-weight } \\
\text { RRR (95\% CI) }\end{array}$ & $\begin{array}{c}\text { Over-weight } \\
\text { RRR (95\% CI) }\end{array}$ \\
\hline \multicolumn{5}{|l|}{ Gender } \\
\hline Male & 1.00 & 1.00 & 1.00 & 1.00 \\
\hline Female & $1.29(1.17-1.42)$ & $1.40(1.29-1.52)$ & $1.38(1.11-1.71)$ & $1.35(1.12-1.63)$ \\
\hline \multicolumn{5}{|l|}{ Wealth quintile } \\
\hline Lowest & 1.00 & 1.00 & 1.00 & 1.00 \\
\hline Second lowest & $0.85(0.74-0.97)$ & $1.25(1.08-1.43)$ & $0.89(0.77-1.03)$ & $1.07(0.93-1.24)$ \\
\hline Middle & $0.76(0.66-0.87)$ & $1.58(1.38-1.81)$ & $0.81(0.69-0.94)$ & $1.26(1.10-1.46)$ \\
\hline Second highest & $0.67(0.58-0.78)$ & $2.23(1.95-2.55)$ & $0.77(0.65-0.91)$ & $1.67(1.44-1.94)$ \\
\hline Highest & $0.48(0.40-0.57)$ & $3.55(3.10-4.05)$ & $0.68(0.55-0.84)$ & $2.27(1.93-2.68)$ \\
\hline \multicolumn{5}{|l|}{ Food availability in last year } \\
\hline Always/sometimes shortage & 1.00 & 1.00 & 1.00 & 1.00 \\
\hline No shortage/no excess & $0.85(0.76-0.96)$ & $1.69-1.52-1.88)$ & $1.01(0.89-1.15)$ & $1.23(1.09-1.38)$ \\
\hline Excess & $0.77(0.68-0.88)$ & $2.25(2.01-2.53)$ & $0.99(0.86-1.17)$ & $1.29(1.12-1.47)$ \\
\hline \multicolumn{5}{|l|}{ Age } \\
\hline $35-39$ years & 1.00 & 1.00 & 1.00 & 1.00 \\
\hline $40-49$ years & $1.12(0.95-1.32)$ & $0.97(0.86-1.32)$ & $1.05(0.87-1.15)$ & $1.01(0.89-1.15)$ \\
\hline $50-59$ years & $1.49(1.27-1.74)$ & $0.89(0.80-1.00)$ & $1.34(1.13-1.58)$ & $0.91(0.80-1.04)$ \\
\hline $60-69$ years & $1.79(1.53-2.10)$ & $0.75(0.67-0.85)$ & $1.62(1.36-1.93)$ & $0.82(0.71-0.94)$ \\
\hline 70 years \& more & $2.61(2.20-3.10)$ & $0.69(0.59-0.80)$ & $2.32(1.89-2.86)$ & $0.77(0.64-0.93)$ \\
\hline \multicolumn{5}{|l|}{ Education } \\
\hline No education & 1.00 & 1.00 & 1.00 & 1.00 \\
\hline $1-4$ years of schooling & $0.69(0.58-0.83)$ & $1.16(1.01-1.33)$ & $0.83(0.69-1.00)$ & $0.99(0.86-1.56)$ \\
\hline $5-9$ years of schooling & $0.64(0.54-0.75)$ & $1.67(1.48-1.89)$ & $0.89(0.75-1.06)$ & $1.20(1.05-1.38)$ \\
\hline$\geq 10$ years of schooling & $1.07(0.94-1.21)$ & $0.93(0.83-1.04)$ & $1.03(0.90-1.17)$ & $0.90(0.80-1.02)$ \\
\hline
\end{tabular}


Table 5 Demographic, Socio-economic and Lifestyle factors associated with under- and over-weight in study population-Continued

\begin{tabular}{|c|c|c|c|c|}
\hline & \multicolumn{2}{|c|}{ Unadjusted analysis } & \multicolumn{2}{|c|}{ Adjusted analysis } \\
\hline & $\begin{array}{l}\text { Under-weight } \\
\text { RRR (95\% CI) }\end{array}$ & $\begin{array}{c}\text { Over-weight } \\
\text { RRR (95\% CI) }\end{array}$ & $\begin{array}{l}\text { Under-weight } \\
\text { RRR (95\% CI) }\end{array}$ & $\begin{array}{c}\text { Over-weight } \\
\text { RRR (95\% CI) }\end{array}$ \\
\hline \multicolumn{5}{|l|}{ Marital status } \\
\hline Currently single & 1.00 & 1.00 & 1.00 & 1.00 \\
\hline Currently married & $0.68(0.60-0.77)$ & $1.22(1.09-1.36)$ & $0.99(0.86-1.15)$ & $1.32(1.16-1.51)$ \\
\hline \multicolumn{5}{|l|}{ Occupation } \\
\hline Agriculture & 1.00 & 1.00 & 1.00 & 1.00 \\
\hline Formal job & $0.54(0.41-0.71)$ & $2.11(1.75-2.56)$ & $0.76(0.57-1.01)$ & $1.52(1.24-1.85)$ \\
\hline Business & $0.53(0.42-0.67)$ & $2.29(1.93-2.70)$ & $0.69(0.54-0.88)$ & $1.77(1.45-2.12)$ \\
\hline Laborer & $0.85(0.70-1.04)$ & $0.59(0.48-0.73)$ & $0.81(0.66-0.99)$ & $0.79(0.64-0.99)$ \\
\hline Home maker & $1.04(0.89-1.21)$ & $1.91(1.67-2.19)$ & $0.95(0.75-1.21)$ & $1.31(1.05-1.64)$ \\
\hline Unemployed & $1.22(0.96-1.56)$ & $1.41(1.12-1.77)$ & $0.96(0.74-1.25)$ & $1.25(0.98-1.59)$ \\
\hline Others & $1.38(1.15-1.65)$ & $1.23(1.03-1.47)$ & $1.06(0.86-1.30)$ & $1.25(1.03-1.52)$ \\
\hline \multicolumn{5}{|l|}{ Residence } \\
\hline Rural & 1.00 & 1.00 & 1.00 & 1.00 \\
\hline Urban & $0.47(0.38-0.59)$ & $1.48(1.31-1.67)$ & $0.66(0.52-0.84)$ & $0.89(0.76-1.04)$ \\
\hline \multicolumn{5}{|l|}{ Meat/Fish consumption } \\
\hline 0-2 days per week & 1.00 & 1.00 & 1.00 & 1.00 \\
\hline 3-4 days per week & $0.83(0.71-0.96)$ & $1.18(1.01-1.38)$ & $0.89(0.76-1.04)$ & $0.92(0.78-1.08)$ \\
\hline 5-7 days per week & $0.63(0.54-0.72)$ & $1.95(1.69-2.25)$ & $0.76(0.65-0.90)$ & $1.01(0.86-1.19)$ \\
\hline \multicolumn{5}{|l|}{ Vegetable consumption } \\
\hline Does not consume daily & 1.00 & 1.00 & 1.00 & 1.00 \\
\hline Consume daily & $0.82(0.73-0.92)$ & $1.55(1.39-1.72)$ & $0.93(0.82-1.05)$ & $1.09(0.97-1.22)$ \\
\hline \multicolumn{5}{|l|}{ Fruits consumption } \\
\hline 0 day per week & 1.00 & 1.00 & 1.00 & 1.00 \\
\hline 1-2 days per week & $0.77(0.69-0.86)$ & $1.61(1.47-1.76)$ & $0.87(0.77-0.97)$ & $1.28(1.16-1.41)$ \\
\hline 3-7 days per day & $0.61(0.52-0.72)$ & $2.53(2.26-2.82)$ & $0.76(0.64-0.92)$ & $1.61(1.41-1.83)$ \\
\hline \multicolumn{5}{|l|}{ Extra salt intake } \\
\hline Never & 1.00 & 1.00 & 1.00 & 1.00 \\
\hline Sometimes & $1.05(0.91-1.21)$ & $0.88(0.79-0.99)$ & $1.00(0.86-1.17)$ & $0.90(0.80-1.02)$ \\
\hline Always & $1.04(0.90-1.19)$ & $0.85(0.76-0.87)$ & $0.96(0.83-1.11)$ & $0.93(0.83-1.04)$ \\
\hline \multicolumn{5}{|l|}{ Daily sleep hours } \\
\hline Less than $7 \mathrm{~h}$ & 1.00 & 1.00 & 1.00 & 1.00 \\
\hline $7-9 \mathrm{~h}$ & $1.04(0.89-1.21)$ & $0.90(0.80-1.02)$ & $1.06(0.90-1.24)$ & $0.97(0.85-1.10)$ \\
\hline More than $9 \mathrm{~h}$ & $1.14(0.93-1.39)$ & $1.14(0.97-1.34)$ & $1.15(0.93-1.42)$ & $1.18(0.99-1.40)$ \\
\hline \multicolumn{5}{|l|}{ Smoking habit } \\
\hline Never smoker & 1.00 & 1.00 & 1.00 & 1.00 \\
\hline Ever smoker & $1.01(0.91-1.12)$ & $0.59(0.54-0.65)$ & $1.32(1.14-1.52)$ & $0.76(0.68-0.86)$ \\
\hline \multicolumn{5}{|l|}{ Smokeless tobacco use } \\
\hline Never user & 1.00 & 1.00 & 1.00 & 1.00 \\
\hline Ever user & $1.26(1.13-1.40)$ & $0.78(0.72-0.85)$ & $0.99(0.88-1.12)$ & $0.95(0.86-1.04)$ \\
\hline \multicolumn{5}{|l|}{ Physical exercise } \\
\hline No exercise & 1.00 & 1.00 & 1.00 & 1.00 \\
\hline$\leq 3 \mathrm{~h}$ exercise per week & $0.81(0.73-0.91)$ & $1.01(0.92-1.11)$ & $0.89(0.79-1.00)$ & $0.98(0.89-1.09)$ \\
\hline$>3 \mathrm{~h}$ exercise per week & $0.83(0.72-0.96)$ & $0.78(0.69-0.89)$ & $1.01(0.87-1.19)$ & $0.92(0.80-1.05)$ \\
\hline
\end{tabular}

\section{DISCUSSIONS}

In this study, we reported the prevalence of underweight $(\mathrm{BMI}<18.5)$ and overweight $(\mathrm{BMI} \geq 23)$ in a nationally representative sample of adult population ( $\geq 35$ years) including both males and females. The findings show presence of a dual burden of undernutrition and overnutrition in the country with remarkably higher prevalence of overweight than underweight, $33.74 \%$ and $18.14 \%$ respectively. Present study shows a significant reduction in prevalence of underweight since 2011 (reported by Biswas and et al.), from 30.4\% to $18.14 \%$ and a remarkable increase in prevalence of overweight, from $23.5 \%$ to $33.74 \%$ [4]. Comparison of nutritional status of adult population with other studies is challenging due to multiple reasons including differences in study time, characteristics of study population and use of different BMI cut points. However, Biswas et al. analyzed data from Bangladesh Demographic and Health Survey of 2011 which is a nationally representative survey, included both males and females of $\geq 35$ years of age and used the Asian population specific cut-points of BMI for classification. Our study is also a nationally representative survey that included similar population and we used the same cut offs. Another study from Bangladesh that included women of age 18 to 49 years reported prevalence of overweight $(\mathrm{BMI} \geq 25) 18.0 \%$ using cut-points of 25, which is very similar to our results [26]. A recent study from South India in adult population of $>19$ years of age reported prevalence of underweight $22.7 \%$ and prevalence of overweight/obese (BMI $\geq 23$ ) 37.0\% [27]. Another study of Sri Lankan male population of age 16-72 years, prevalence of overweight/obese (BMI $\geq 23$ ) was found to be $44.1 \%$ [15]. 
Our study shows that females are at greater risk of both underweight and overweight compared to males. Previous studies also reported that females are more likely to be overweight compared to males. Biswas et al. reported females are nearly three times more prone to be overweight compared to males [4]. Another study from Iranian population also reported higher odds of overweight among women compared to men (OR:2.05; 95\% CI:1.15-3.65) [28]. Higher risk of underweight in females is also consistent with similar studies. The study conducted by Biswas et al. reports unadjusted odds ratio of 1.45 (1.27-1.66) for females compared to males [4]. Another study conducted among Nigerian young adult population reported that prevalence of both underweight and overweight were higher among females [29].

We found that higher wealth quintiles are positively associated with overweight while negatively associated with underweight. These are consistent with findings with other studies [4,27]. Older age significantly increased the risk of being underweight, while decreased the risk of being overweight, which is in line with the findings from similar studies [4]. We did not find significant association between nutritional status and educational level in the adjusted model as opposed to reported in previous studies $[4,16,30]$. However, significant association is observed in the unadjusted analysis. This findings of no association with education level in the adjusted analysis is perhaps due to the fact that education level works through dietary practice and other lifestyle factors and we adjusted for all these factors in our multivariable analysis [31]. We find that currently married people are more likely to be overweight compared to single people in the adjusted analysis but marital status does not have significant association with underweight. This positive association between currently married people and overweight is consistent with findings in previous studies [32,33]. We did not find any significant association between marital status and underweight in the adjusted analysis as found in one previous study conducted in elderly people in Portuguese [34]. Urban residence decreased risk of being underweight and overweight compared to rural residence. Similar findings were found for underweight in other studies in Bangladesh where compared to urban people, rural people were at higher risk of being underweight [4]. Although the above stated study found increased risk of being overweight in urban population, we found similar association in crude analysis but decreased risk in the adjusted analysis. We argue that this higher risk of overweight in urban population is likely to mediate through dietary habit and other lifestyle behaviors. As in our study we adjusted all dietary habits, therefore, we did not find significant positive association in the adjusted analysis. More frequent protein consumption (meat/fish) decreased the risk of being underweight but no significant association between overweight and protein consumption was found in our study. One problem in this study is that we did not collect data separately on meat and fish, which might have shown different results. One study in Vietnamese rural adult population that collected data separately on meat and fish consumption, found no signification association of nutritional status with meat consumption but found significant association with fish consumption [35]. In our study higher frequency of fruits consumption significantly decreased risk of being underweight but increased risk of being overweight. However, we did not find significant association for vegetable consumptions. Comparison of these results with other studies is challenging due to wide variation in data collection methods. One study that collected combined data on fruits and vegetables found significant association in crude analysis but no association in multivariable analysis [27]. Another study in Vietnam collecting data on fruits consumption only showed significant association in crude analysis [35]. We did not find any significant association between extra salt intake and overweight as found in previous studies $[36,37]$. Our study did not find significant association between sleep duration and underweight or overweight. Earlier studies found significant association between shorter and longer sleep duration with overweight in both cross-sectional as well as longitudinal studies [38,39]. Smokers in our study population were significantly at higher risk of being underweight and lower risk of being overweight. These findings are in agreement with other studies in India and China $[27,40,41]$ as well as in the US $[42,43]$. The reasons for this type of association may be direct effect of tobacco on appetite and other aspects of physiology or indirect effect on reduced purchasing power for food and therefore quality of diet [44].

Our study has several strengths. This is a large nationally representative population-based study including male/female and rural/urban respondents. We collected full range of demographic, socio-economic, lifestyle and dietary factors from respondent likely to be associated with underweight and overweight. We conducted multivariable multinomial logistics regression to identify the determinants of both underweight and overweight in the same model. There are few limitations of this study as well. This is a cross-sectional study where information on outcome and exposure variables were collected at the same point in time. Thus, the association between various factors and nutritional status may not be causal in nature. Majority of the exposure variables were self-reported likely to induce measurement error. However, we believe that measurement errors are non-differential in nature due to extensive training of data collectors and strict quality control in data collection procedures resulting in underestimation of associations.

\section{CONCLUSIONS}

We report existence of a double burden of underweight and overweight in Bangladesh adult population. Both overweight and underweight results in a wide range of morbidity and mortality that impose a huge negative impact on socio-economic development. A number of socio-economic, demographic, lifestyle and dietary factors are significantly associated with underweight and overweight in adult population of Bangladesh. Population level impact of these factors should be examined to design suitable public health and nutrition interventions to address this dual challenge.

\section{DATA STATEMENT}

The data is available on request.

\section{CONFLICT OF INTEREST}

None. 


\section{ACKNOWLEDGMENTS}

This research activity was supported by the Strategic Partnership Agreement (SPA) with AusAID and UKAID. BRAC acknowledges with gratitude the commitment of the AusAID and UKAID to its research efforts. We thank the respondents who gave their time generously to provide information as well as the field data collection team and data management staff who worked tirelessly.

\section{APPENDIX A. SUPPLEMENTARY DATA}

Supplementary data associated with this article can be found, in the online version, at https://doi.org/10.2991/j.jegh.2018.03.002.

\section{REFERENCES}

[1] Popkin BM, Adair LS, Ng SW. Global nutrition transition and the pandemic of obesity in developing countries. Nutr Rev 2012;70;3-21.

[2] Mascie-Taylor N. Is Bangladesh going through an epidemiological and nutritional transition? Collegium Antropologicum 2012;36;1155-9.

[3] Khan SH, Talukder SH. Nutrition transition in Bangladesh: is the country ready for this double burden. Obesity Rev: Off J Int Assoc Study Obesity 2013;14;126-33.

[4] Biswas T, Garnett SP, Pervin S, Rawal LB. The prevalence of underweight, overweight and obesity in Bangladeshi adults: data from a national survey. PLoS One 2017;12;e0177395.

[5] Nube M, Van Den Boom GJ. Gender and adult undernutrition in developing countries. Ann Hum Biol 2003;30;520-37.

[6] Ng M, Fleming T, Robinson M, Thomson B, Graetz N, Margono $\mathrm{C}$, et al. Global, regional, and national prevalence of overweight and obesity in children and adults during 1980-2013: a systematic analysis for the Global Burden of Disease Study 2013. Lancet 2014;384;766-81.

[7] Sairenchi T, Iso H, Irie F, Fukasawa N, Ota H, Muto T. Underweight as a predictor of diabetes in older adults: a large cohort study. Diabetes Care 2008;31;583-4.

[8] Zheng W, McLerran DF, Rolland B, Zhang X, Inoue M, Matsuo K, et al. Association between body-mass index and risk of death in more than 1 million Asians. N Engl J Med 2011;364;719-9.

[9] Ni Mhurchu C, Rodgers A, Pan WH, Gu DF, Woodward M. Asia Pacific cohort studies C. body mass index and cardiovascular disease in the Asia-Pacific Region: an overview of 33 cohorts involving 310000 participants. Int J Epidemiol 2004;33; 751-8.

[10] World Health Organization. Global Health Risks: Mortality and Burden of Disease Attributable to Selected Major Risks, Geneva: World Health Organization; 2009.

[11] Danaei G, Ding EL, Mozaffarian D, Taylor B, Rehm J, Murray CJ, et al. The preventable causes of death in the United States: comparative risk assessment of dietary, lifestyle, and metabolic risk factors. PLoS Med 2009;6;e1000058.

[12] Ewing R, Schmid T, Killingsworth R, Zlot A, Raudenbush S. Relationship between urban sprawl and physical activity, obesity, and morbidity. American J Health Promotion 2003;18;47-57.

[13] Kleiser C, Rosario AS, Mensink GB, Prinz-Langenohl R, Kurth B. Potential determinants of obesity among children and adolescents in Germany: results from the cross-sectional KiGGS Study. BMC Public Health 2009;9;46.

[14] Janssen I, Katzmarzyk PT, Boyce WF, Vereecken C, Mulvihill C, Roberts C, et al. Comparison of overweight and obesity prevalence in school-aged youth from 34 countries and their relationships with physical activity and dietary patterns. Obesity Rev 2005;6;123-32.

[15] Jayawardana NWIA, Jayalath WATA, Madhujith WMT, Ralapanawa U, Jayasekera RS, Alagiyawanna SASB, et al. Lifestyle factors associated with obesity in a cohort of males in the central province of Sri Lanka: a cross-sectional descriptive study. BMC Public Health 2017;17;27.

[16] Subramanian SV, Smith GD. Patterns, distribution, and determinants of under- and overnutrition: a population-based study of women in India. Am. J Clin Nutr 2006;84;633-40.

[17] Hartung C, Lerer A, Anokwa Y, Tseng C, Brunette W, Borriello G. Open data kit: tools to build information services for developing regions. In: Proceedings of the 4th ACM/IEEE International Conference on Information and Communication Technologies and Development, ACM. 2010;18.

[18] Yorkin M, Spaccarotella K, Martin-Biggers J, Quick V, ByrdBredbenner C. Accuracy and consistency of weights provided by home bathroom scales. BMC Public Health 2013;13;1194.

[19] Zabeen B, Tayyeb S, Naz F, Ahmed F, Rahman M, Nahar J, et al. Prevalence of obesity and central obesity among adolescent girls in a district school in Bangladesh. Indian J Endocrinol Metab 2015;19;649-52.

[20] WHO Expert Consultation. Appropriate body-mass index for Asian populations and its implications for policy and intervention strategies. Lancet 2004;363;157-63.

[21] Nagai M, Tomata Y, Watanabe T, Kakizaki M, Tsuji I. Association between sleep duration, weight gain, and obesity for long period. Sleep Med 2013;14;206-10.

[22] Yunus FM, Khan S, Akter T, Jhohura FT, Reja S, Islam A, et al. How many hours do people sleep in Bangladesh? a countryrepresentative survey. J Sleep Res 2016;14.

[23] NSF. National sleep foundation recommends new sleep times. https://sleepfoundation.org/media-center/press-release/nationalsleep-foundation-recommends-new-sleep-times; 2015 [accessed 19/4/2016.2016].

[24] Vyas S, Kumaranayake L. Constructing socio-economic status indices: how to use principal components analysis. Health Policy Plann 2006;21;459.

[25] StatCorp. Stata Statistical Software: Release 12 College Station, TX: Stata Corporation; 2011.

[26] Sarma H, Saquib N, Hasan MM, Saquib J, Rahman AS, Khan JR, et al. Determinants of overweight or obesity among ever-married adult women in Bangladesh. BMC Obes 2016;3;13.

[27] Little M, Humphries S, Patel K, Dewey C. Factors associated with BMI, underweight, overweight, and obesity among adults in a population of rural south India: a cross-sectional study. BMC Obes 2016;3;12.

[28] Nikooyeh B, Abdollahi Z, Salehi F, Nourisaeidlou S, Hajifaraji M, Zahedirad M, et al. Prevalence of obesity and overweight and its associated factors in urban adults from West Azerbaijan, Iran: the national food and nutritional surveillance program (NFNSP). Nutr Food Sci Res 2016;3;21-6.

[29] Ogunlade O, Asafa M. Pattern and prevalence of underweight, overweight and obesity among young adult Nigerians. Am J Biomed Life Sci 2015;3;12-15. 
[30] Letamo G, Navaneetham K. Prevalence and determinants of adult under-nutrition in Botswana. PLoS One 2014;9;e102675.

[31] Kriaučionienè V, Petkevičienè J, Klumbienė J. Dietary patterns and their association with sociodemographic factors in Lithuanian adult population. Medicina 2008;44;799-804.

[32] Sen J, Mondal N, Dutta S. Factors affecting overweight and obesity among urban adults: a cross-sectional study. Epidemiol Biostat Public Health 2013;10.

[33] Jeffery RW, Rick AM. Cross-sectional and longitudinal associations between body mass index and marriage-related factors. Obesity 2002;10;809-15.

[34] Santos A, Amaral T, Borges N. Undernutrition and associated factors in a Portuguese older adult community. Revista de Nutrição 2015;28;231-40.

[35] Kim SH, Hwang JY, Kim MK, Chung HW, Nguyet TT, Kim WY. Dietary factors related to body weight in adult Vietnamese in the rural area of Haiphong, Vietnam: the Korean Genome and Epidemiology Study (KoGES). Nutr Res Pract 2010;4;235-42.

[36] Yi SS, Kansagra SM. Associations of sodium intake with obesity, body mass index, waist circumference, and weight. Am J Prev Med 2014;46;e53-5.

[37] Yoon YS, Oh SW. Sodium density and obesity; the Korea National Health and Nutrition Examination Survey 2007-2010. Eur J Clin Nutr 2013;67;141-6.
[38] Yiengprugsawan V, Banwell C, Seubsman SA, Sleigh AC. Thai Cohort Study T. Short sleep and obesity in a large national cohort of Thai adults. BMJ Open 2012;2;e000561.

[39] Chaput J, Després J, Bouchard C, Tremblay A. The association between sleep duration and weight gain in adults: a 6-year prospective study from the Quebec Family Study. Sleep 2008;31; $517-23$.

[40] Wang Q. Smoking and body weight: evidence from China health and nutrition survey. BMC Public Health 2015;15;1238.

[41] Pednekar MS, Gupta PC, Shukla HC, Hebert JR. Association between tobacco use and body mass index in urban Indian population: implications for public health in India. BMC Public Health 2006;6;70.

[42] Plurphanswat N, Rodu B. The association of smoking and demographic characteristics on body mass index and obesity among adults in the U.S., 1999-2012. BMC Obes 2014;1;18.

[43] Albanes D, Jones D, Micozzi MS, Mattson ME. Associations between smoking and body weight in the US population: analysis of NHANES II. Am J Public Health 1987;77;439-44.

[44] Rani M, Bonu S, Jha P, Nguyen SN, Jamjoum L. Tobacco use in India: prevalence and predictors of smoking and chewing in a national cross sectional household survey. Tobacco Control $2003 ; 12 ; \mathrm{e} 4$. 